\title{
Impedance of a Circular Loop in an Infinite Conducting Medium
}

\author{
Martin B. Kraichman \\ Contribution from U.S. Naval Ordnance Laboratory, White Oak, Silver Spring, Md.
}

(Received February 12, 1962)

\begin{abstract}
Expressions are derived for the resistance and reactance of a circular loop of thinly insulated wire which carries a uniform current and is immersed in a conducting medium. The result for the resistance is combared with that known for a circular loop in a spherical insulating cavity.
\end{abstract}

\section{Introduction}

There is a current interest in the use of loops for transmitting and receiving electromagnetic energy in a dissipative medium. Expressions for the impedance of a loop in such a medium are of value in designing radiating and receiving systems. The resistance of a circular loop in a conducting medium has been discussed by Moore [1951]. In the present paper, expressions are derived for both the resistance and reactance of a circular loop of thinly insulated wire which carries a uniform current and is surrounded by an infinite, homogeneous, conducting medium. These expressions are valid if the wavelength in the conducting medium is large compared with the diameter of the loop and if the displacement current in the medium is negligible. The result for the resistance is compared with that of Wait [1957] for a circular loop in a spherical insulating cavity.

\section{Impedance Integral}

An integral expression for the impedance of a single turn loop carrying uniform current in free space is given by Schelkunoff [1943]. For a loop immersed in a conducting medium, the propagation constant in this expression will be complex. Referring to figure 1, the expression reads

where

$$
Z=\frac{j \omega \mu}{4 \pi} \iint \frac{e^{-\gamma r_{12}}}{r_{12}} \cos \psi d s_{1} d s_{2}
$$

$\omega=$ angular frequency of loop current

$\mu=$ permeability of conducting medium

$\gamma=$ complex propagation constant

$d s_{1}=$ differential element of length along wire center

$d s_{2}=$ differential element of length along inner surface of wire

$r_{12}=$ distance between differential elements $d s_{1}$ and $d s_{2}$.

The loop wire is assumed to have a coating of insulation which confines the impressed current to the wire. If this insulation is thin, the results presented here for negligible insulation thickness are applicable. In calculating the impedance, the current in the loop may be assumed to be concentrated along the center of the wire. This is a good approximation if the wire diameter is much smaller than the loop diameter and if the circular symmetry of the current distribution in the wire is not greatly disturbed because of the proximity effect.

If the displacement current in the conducting medium is neglected, the complex propagation constant may be written as

$$
\gamma=(j \omega \mu \sigma)^{1 / 2}=(i+j) \beta, \quad \text { where } \quad \beta=\left(\frac{\omega \mu \sigma}{2}\right)^{1 / 2}
$$




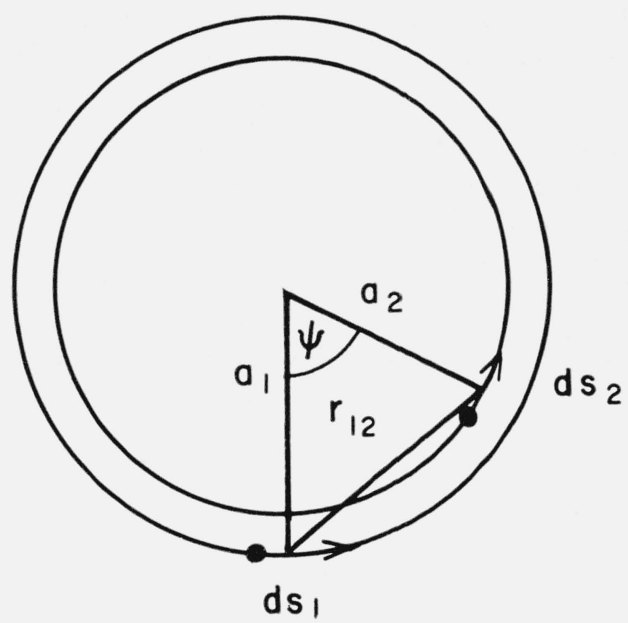

Figure 1. Circular loop, showing wire axis and wire inner surface.

and $\sigma$ is the conductivity of the dissipative medium. The real and imaginary parts of the impedance in eq (1) may then be written as

and

$$
R=\frac{\omega \mu}{4 \pi} \iint \frac{e^{-\beta^{r_{12}}}}{r_{12}} \sin \beta r_{12} \cos \psi d s_{1} d s_{2}
$$

$$
X=\frac{\omega \mu}{4 \pi} \iint \frac{e^{-\beta r_{12}}}{r_{12}} \cos \beta r_{12} \cos \psi d s_{1} d s_{2},
$$

respectively, where $R$ is the resistance and $X$ is the inductive reactance. The above expression for the resistance of a loop represents external losses and does not include internal losses in the loop wire. Similarly, the above expression for the reactance represents only the contribution of external inductance. The second integration in eq (3) should be along a curve on the inner surface of the wire so that only flux external to the wire is enclosed. The internal resistance and inductance can be computed separately from well known formulas [Ramo and Whinnery, 1953].

\section{Evaluation of Impedance Integral}

The integrands in eqs (2) and (3) may be expanded in a power series and the expressions for the resistance and reactance written as

and

$$
R=\frac{\beta \omega \mu}{4 \pi} \iint\left[1-\beta r_{12}+\frac{1}{3}\left(\beta r_{12}\right)^{2}-\frac{1}{30}\left(\beta r_{12}\right)^{4}+\ldots\right] \cos \psi d s_{1} d s_{2}
$$

$$
X=\frac{\beta \omega \mu}{4 \pi} \iint\left[\frac{1}{\beta r_{12}}-1+\frac{1}{3}\left(\beta r_{12}\right)^{2}-\frac{1}{6}\left(\beta r_{12}\right)^{3}+\ldots\right] \cos \psi d s_{1} d s_{2} .
$$

No great error is made in calculating the resistance if the second integration is also performed along the center of the wire. The error involved in shifting the second path of integration from the inner surface to the center of the wire is greatest for small values of $r_{12}$, and for these values, the integrand is not strongly dependent on $r_{12}$. The greatest contribution to the integrand of eq (5), however, comes from small values of $r_{12}$.

\subsection{Evaluation of Resistance Integral}

To calculate the external resistance of a circular, single turn loop in a conducting medium, the integration indicated in eq (4) may be performed by referring to figure 2. The circle of radius $a$ represents the wire axis of the loop. With $d s_{1}=a d \theta, d s_{2}=a d \psi$, and $r_{12}=2 a$ sin $\psi / 2$, the resistance is given by 


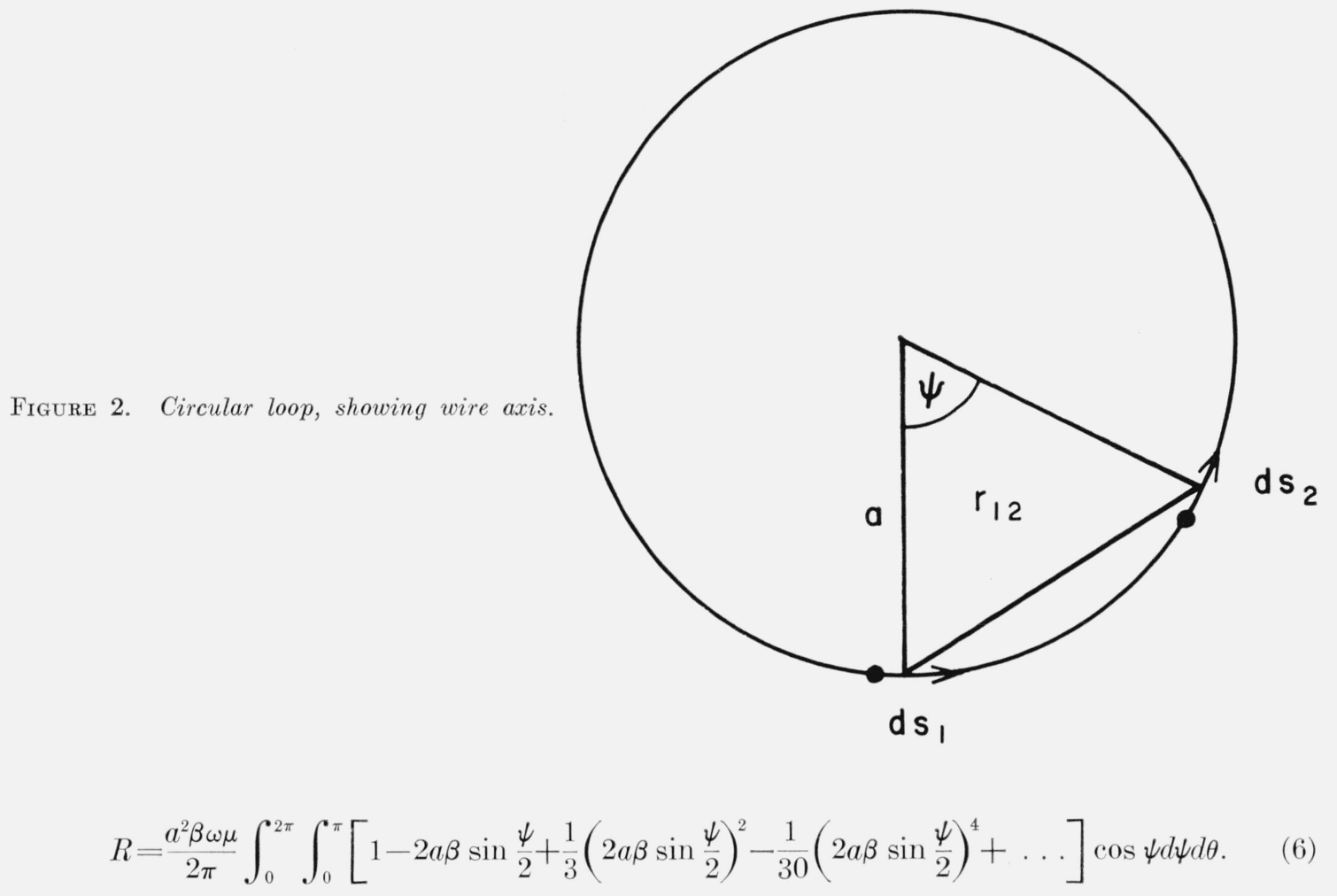

Term by term integration results in

$$
R=\omega \mu a\left[\frac{4}{3}(\beta a)^{2}-\frac{\pi}{3}(\beta a)^{3}+\frac{2 \pi}{15}(\beta a)^{5}-\ldots\right] .
$$

For a loop of $N$ turns, the resistance would be multiplied by the factor $\Lambda^{2}$.

The radiation resistance of a single turn, circular loop in air is given by

$$
R_{\mathrm{air}}=\frac{\pi}{6} \frac{\omega^{4} \mu a^{4}}{c^{3}}
$$

where $c$ is the velocity of light in free space. Since the displacement current in the conducting medium is quite small compared with the conduction current, the radiation resistance of a loop in air is very much smaller than the resistance of that loop when immersed in a conducting medium.

\subsection{Evaluation of Reactance Integral}

Using figure 1, the external reactance of a single turn, circular loop may be calculated by performing the integration indicated in eq (5). The wire axis and the wire inner surface are represented by concentric circles of radius $a_{1}$ and $a_{2}$, respectively. With $d s_{1}=a_{1} d \theta$ and $d s_{2}=a_{2} d \psi$, eq (5) may be written as

$$
X=\frac{\beta \omega \mu a_{1} a_{2}}{2 \pi} \int_{0}^{2 \pi} \int_{0}^{\pi}\left[\frac{1}{\beta r_{12}}-1+\frac{1}{3}\left(\beta r_{12}\right)^{2}-\frac{1}{6}\left(\beta r_{12}\right)^{3}+\ldots\right] \cos \psi d \theta .
$$

The contributions to the integral of the second and succeeding terms in the integrand above are changed little by letting $a_{1}=a_{2}=a$ and $r_{12}=2 a \sin \psi / 2$. In the first term, however, $a_{1}$ must be distinguished from $a_{2}$. Using the law of cosines, $r_{12}^{2}=a_{1}^{2}+a_{2}^{2}-2 a_{1} a_{2} \cos \psi$. Equation (9) may then be written as 


$$
\begin{aligned}
& X=\frac{\omega \mu a_{1} a_{2}}{2 \pi} \int_{0}^{2 \pi} \int_{0}^{\pi} \frac{\cos \psi d \psi d \theta}{\left[a_{1}^{2}+a_{2}^{2}-2 a_{1} a_{2} \cos \psi\right]^{1 / 2}} \\
& \quad+\frac{\beta \omega \mu a^{2}}{2 \pi} \int_{0}^{2 \pi} \int_{0}^{\pi}\left[-1+\frac{1}{3}\left(2 a \beta \sin \frac{\psi}{2}\right)^{2}-\frac{1}{6}\left(2 a \beta \sin \frac{\psi}{2}\right)^{3}+\ldots\right] \cos \psi d \psi d \theta .
\end{aligned}
$$

By substituting $\psi=\pi+2 \phi$ and $k^{2}=\frac{4 a_{1} a_{2}}{\left(a_{1}+a_{2}\right)^{2}}$, the first integral in eq (10) may be written as

$$
I_{1}=\frac{\omega \mu a_{1} a_{2}}{2 \pi}\left[\left(\frac{2 \pi\left(a_{1}+a_{2}\right)}{a_{1} a_{2}}-\frac{4 \pi}{a_{1}+a_{2}}\right) \int_{0}^{\frac{\pi}{2}} \frac{d \phi}{\left(1-k^{2} \sin ^{2} \phi\right)^{1 / 2}}-\frac{2 \pi\left(a_{1}+a_{2}\right)}{a_{1} a_{2}} \int_{0}^{\frac{\pi}{2}}\left(1-k^{2} \sin ^{2} \phi\right)^{1 / 2} d \phi\right] .
$$

The integrals in eq (11) may be recognized as the complete elliptic integrals of the first and second kind respectively. For loops of small wire diameter, $a_{1} \cong a_{2}=a$ and $k^{2} \approx 1$. In this case, the complete elliptic integral of the second kind is approximately equal to unity. Equation (11) then becomes

$$
I_{1}=\omega \mu a[K(k)-2],
$$

where

$$
K(k)=\int_{0}^{\frac{\pi}{2}} \frac{d \phi}{\left(1-k^{2} \sin ^{2} \phi\right)^{1 / 2}}
$$

is the complete elliptic integral of the first kind. The remaining integrals in eq (10) are simple and may be integrated term by term. The expression for the reactance then becomes

$$
X=\omega \mu a\left[K(k)-2-\frac{\pi}{3}(\beta a)^{3}+\frac{4}{15}(\beta a)^{4}-\ldots\right] .
$$

Since the reactance of a single turn, circular coil in air is given by

$$
X_{\mathrm{a} 1 \mathrm{r}}=\omega \mu a[K(k)-2],
$$

equation (13) may be written as

$$
X=X_{\mathrm{a} 1 \mathrm{r}}-\omega \mu a\left[\frac{\pi}{3}(\beta a)^{3}-\frac{4}{15}(\beta a)^{4}+\ldots\right]
$$

where the series of terms on the right side of eq (15) represents a correction due to the conducting medium. For a circular loop of $N$ turns, the reactance would be multiplied by the factor $N^{2}$.

\section{Plot of Results}

Values of the immersion correction terms for the resistance and the reactance are presented in figure 3. These terms are obtained from eqs (7) and (15) and are plotted as the dimensionless quantities $\frac{R_{\text {cor }}}{\omega \mu a}$ and $\frac{X_{\text {cor }}}{\omega \mu a}$ versus $\beta a$. For $\beta a \ll 1$, the immersion correction for the resistance is very nearly $\beta a$ times that for the reactance.

\section{Effect of Spherical Insulating Cavity}

It is of interest to compare the expression for the resistance given by eq (7) with that of Wait [1957] for the resistance of a circular loop in a spherical insulating cavity. The immersion correction for the resistance is given by Wait as 
FIGURE 3. Immersion correction terms for the resistance and reactance of a single turn circular loop.

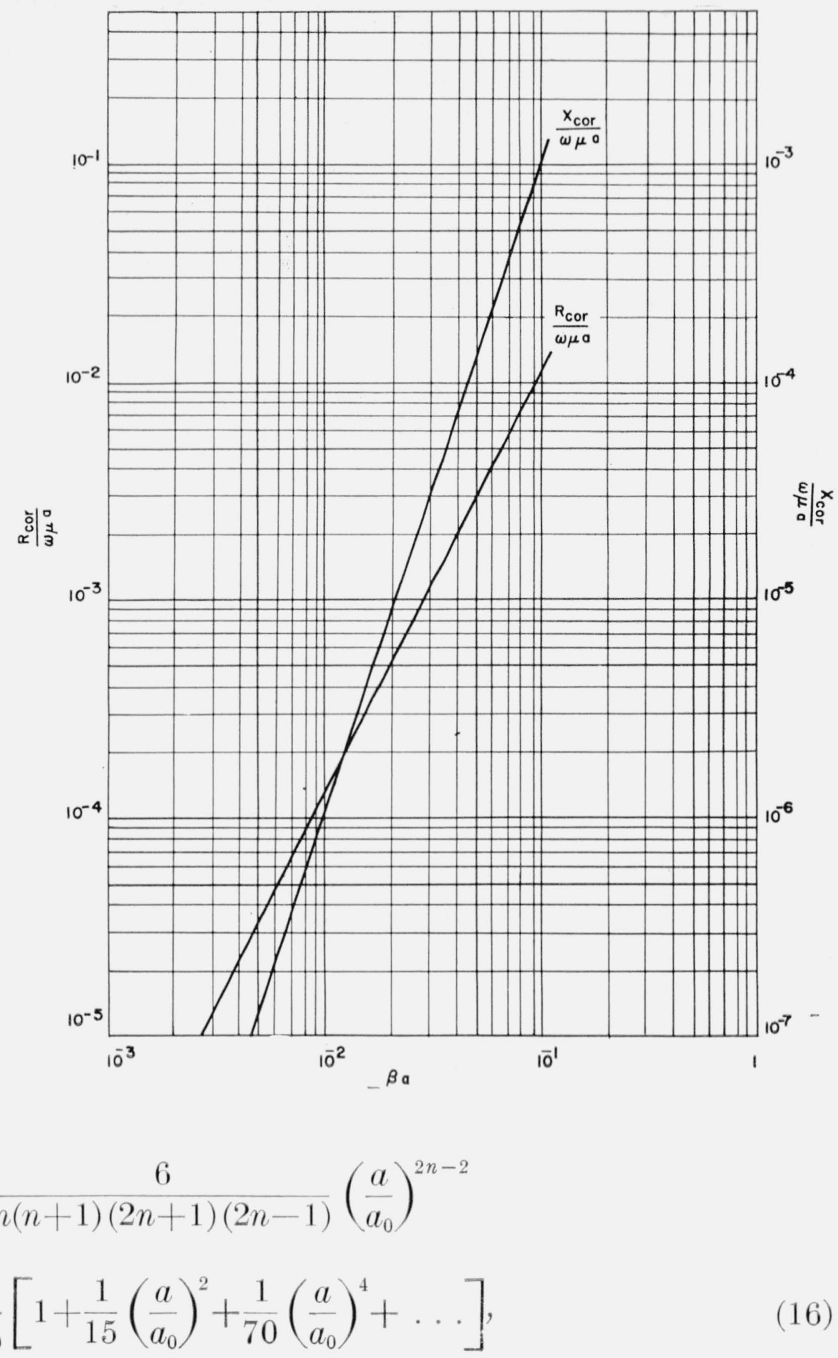

where

$a=$ radius of loop with center at the center of sphere

$a_{0}=$ radius of spherical insulating cavity.

If $a=a_{0}$ and $\beta a \ll 1$, the ratio of the resistance correction given by eq (16) to that given by eq (7) is approximately equal to 0.86 . The close agreement between the two immersion corrections indicates the small eifect of a spherical insulating core on the loop resistance. When $a / a_{0} \ll 1$ and $\beta a \ll 1$, the ratio of the resistance correction terms is approximately equal to $a / a_{0}$. This clearly shows the effectiveness of the spherical insulating cavity in reducing losses.

\section{References}

Moore, R. K., The theory of radio communication between submerged submarines, Ph. D. Thesis, Cornell University (1951).

Ramo, S., and Whinnery, J. R., Fields and waves in modern radio, 2d ed., Chap. 6, p. 247 (John Wiley \& Sons, 1953).

Schelkunoff, S. A., Electromagnetic waves, Chap. 6, p. 145 (D. Van Nostrand Co., 1943).

Wait, J. R., Insulated loop antenna immersed in a conducting medium, J. Research NBS 59, No. 2, $133-137$ (Aug. 1957). 